\title{
EBUS-TBNA for the diagnosis and staging of lung cancer
}

F. J. F. Herth ${ }^{1}$, M. Krasnik², P. Vilmann²

${ }^{1}$ Department of Pneumology and Critical Care Medicine, Thoraxklinik at University Heidelberg, Germany

2 Department of Cardiothoracic Surgery, Gentofte and Department of Surgical Gastroenterology University Hospital, Copenhagen, Denmark

\section{Introductiuon}

Non-small cell lung cancer (NSCLC) usually metastasizes first to hilar and mediastinal lymph nodes. Subsequently, hematogenous metastasis to distant sites may occur. Because survival is inversely correlated with stage, a meticulous staging procedure is required to determine the treatment and prognosis [1,2]. For staging of NSCLC, the TNM classification has been developed, in which $\mathrm{T}$ stands for local tumor extension, $\mathrm{N}$ for lymph node metastasis, and $\mathrm{M}$ for distant metastasis. The lymph node map by Mountain et al., and its revisions are often used for the description of the $\mathrm{N}$ factor of the TNM classification [3]. Mediastinal lymph node staging can be divided into imaging and sampling. Computed tomography (CT), magnetic resonance imaging (MRI), and positron emission tomography (PET) may be used to image mediastinal lymph nodes [4]. Pathologic sampling of suspicious lesions can be performed by mediastinoscopy, thoracoscopy, transthoracic fine-needle aspiration, transbronchial fine-

Correspondence: Prof. Felix JF Herth, Md, FCCP, DSc · Head · Department of Pneumology and Critical Care Medicine - Thoraxklinik, University of Heidelberg · Amalienstr. 5 - 69126 Heidelberg · Germany - Phone: +49-6221-396600 . Fax: +49-6221-396602 · E-mail: Felix.Herth@thoraxklinik-heidelberg.de, www. thoraxklinik-heidelberg.de

Bibliography: Endoscopy 2006; 38 (S1): S101-S105 @ Georg Thieme Verlag KG Stuttgart · New York · ISSN 0013-726X · DOI 10.1055/s-2006-946668 
needle aspiration, and endoscopic ultrasonography with fineneedle aspiration $[4,5]$.

For many years mediastinoscopy has been regarded as the 'gold standard' for staging of the mediastinum but it is invasive, requires a general anaesthetic and is costly. While a standard cervical mediastinoscopy permits access to paratracheal lymph node stations (levels $2 \mathrm{R}, 2 \mathrm{~L}, 4 \mathrm{R}$ and $4 \mathrm{~L}$ ) and the anterior subcarinal lymph node station (level 7), access to the posterior and inferior mediastinum is limited and requires either extended cervical mediastinoscopy or a thoracoscopy [6].

Since 2004 a novel prototype linear array endobronchial ultrasound (EBUS) probe with a fine needle biopsy facility for realtime imaging and aspiration biopsy of paratracheal and parabronchial lymph nodes (stations 2, 3, 4, 7,10 and 11) is available. With this scope direct TBNA under real time convex probe endobronchial ultrasonography (EBUS-TBNA-bronchoscopy) guidance is now possible.

\section{Endobronchial ultrasound}

Endobronchial ultrasound (EBUS) using a radial probe is an imaging technique capable of detecting even small mediastinal lymph nodes [7]. However, imaging alone is inaccurate. Therefore, tissue confirmation of suspected malignant lymphadenopathy is required especially before surgical resection. To obtain tissue confirmation, different invasive and non-invasive methods have been performed.

In particular, endoscopic ultrasound fine-needle aspiration (EUSFNA) is a well-known modality with a high yield for tissue sampling of the mediastinum.

\section{Lymph node staging}

Under favourable conditions lymph nodes can be detected by EBUS down to a size of $2-3 \mathrm{~mm}$ and the internal structure (sinuses and folliculi) as well as small lymph vessels can be analyzed. By endosonographic localization of lymph nodes the results of transbronchial needle aspiration (TBNA) can be significantly improved with a sensitivity up to $85 \%$ [8]. This is especially true for those positions in which reliable landmarks on the CT are missing, e.g. high and low paratracheal localization. Herth et al. [9] investigated the results of an EBUS guided TBNA compared to conventional TBNA. In this randomized study the group could confirm, that the yield of an EBUS guided TBNA is higher than conventional TBNA ( $85 \%$ vs. $66 \%$ ). In an additional analysis of the lymph node stations they showed, that especially in a location without endoscopic landmarks (LN station 2, 3, 4 Mountain scheme) the detection technique is helpful to increase the yield. On the other hand it was also demonstrated, that in case of enlarged subcarinal nodes a conventional TBNA has the same yield as TBNA after EBUS detection [7].

Since the development of the EBUS-TBNA scope the use of miniprobes for guidance of a TBNA is decreasing dramatically.
The flexible ultrasonic bronchoscope (Olympus BF-UC160F-OL8, Tokyo, Japan) (Fig. 1) has an outer diameter of the insertion tube is $6.7 \mathrm{~mm}$, working length is $550 \mathrm{~mm}$ (total lengths $870 \mathrm{~mm}$ ). The instrument has a small curved linear array electronic transducer, length $10 \mathrm{~mm}$, located at the distal end of the endoscope in front of a 30 degree oblique forward viewing fiber-optic lens (angle of view is 80 degree) (Fig. 2). The diameter of the distal end of the endoscope with the transducer is $6.9 \mathrm{~mm}$. The angulation range of the distal end of the endoscope is 160 degrees upward and 90 degrees downward. The endoscope has a biopsy channel of $2 \mathrm{~mm}$. The ultrasonic frequency is $7.5 \mathrm{MHz}$ with a penetration depth of $4-5 \mathrm{~cm}$. The depth of imaging can be adjusted from 2 to $9 \mathrm{~cm}$ with $1 \mathrm{~cm}$ intervals. The scanning direction is parallel to the longitudinal axis of the endoscope with a scanning angle of 50 degree. This enables full ultrasonic monitoring of a needle when inserted via the biopsy channel during scanning. The endoscope can either be connected to an Olympus ultrasound processor (EU C60, Olympus, Tokyo, Japan) or to an Aloka Ultra-

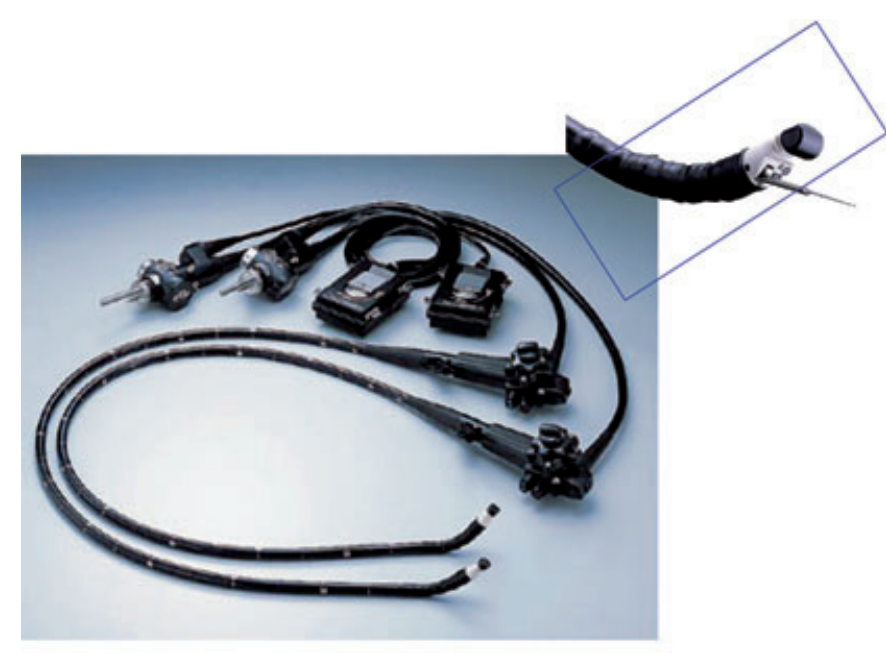

Fig. 1 Tipp of the linear array ultrasonic bronchoscope (BF-UC160FOL8, Olympus Ltd., Tokyo Japan).

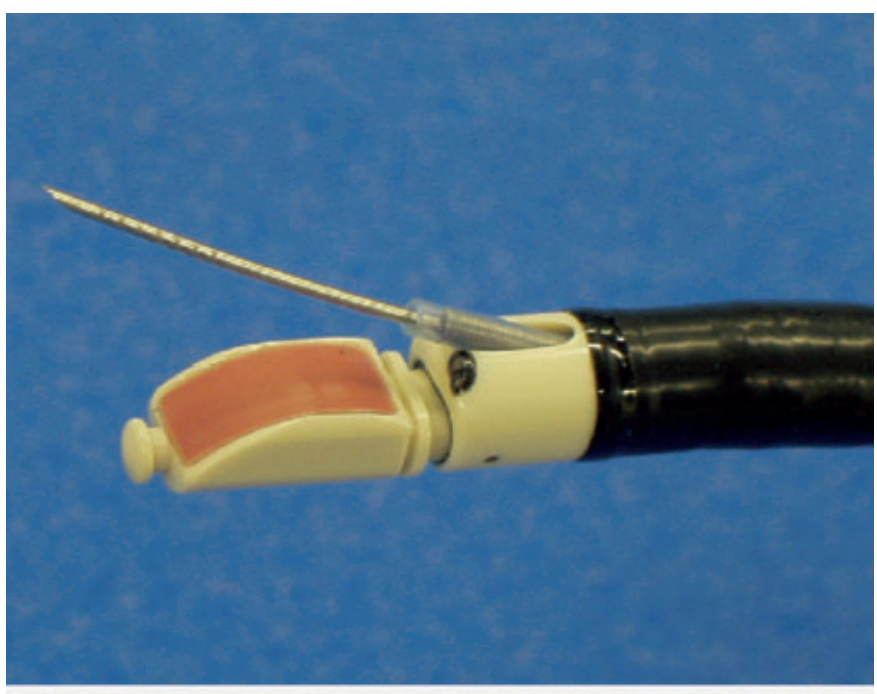

Fig. 2 The needle is extended through the working channel and exits obliquely. 
sound scanner. A balloon, which can be filled with water, can be mounted around the transducer for better ultrasonic coupling with the bronchial wall. The ultrasound images can be frozen and the size of lesions can be measured in two dimensions by the placement of cursors. This system also has the Doppler mode.

A dedicated 22-gauge needle was developed to perform transbronchial aspiration (NA-201SX-4022, Olympus, Tokyo, Japan) (Fig. 3). The inner diameter of this needle is nearly equal to that of a conventional 21-gauge needle which allows sampling of histological cores in some cases. The needle is also equipped with an internal sheath which is withdrawn after passing the bronchial wall, avoiding contamination during EBUS-TBNA. The needle can be visualized through the optics and on the ultrasound image.

\section{Procedure}

The ultrasonic bronchoscope is introduced via an endotracheal tube under visual control or under local anaesthesia to the area of interest, mostly on an outpatient basis. The bronchoscope is inserted orally with additional sedation with usually $2 \mathrm{mg}$ midazolam. Patients must be monitored for ECG, pulse oximetry, and blood pressure without the presence of an anaesthesiologist. Images can be obtained by directly contacting the probe or by attaching a balloon on the tipp and inflating with saline. Since the balloon is designed not to over inflate, it will not occlude the central airway. No experience technical difficulties using the balloon are reproted.

When a lesion is outlined, the needle is introduced via the biopsy channel of the endoscope. Power Doppler examination is used immediately before the biopsy in order to avoid unintended puncture of vessels between the wall of the bronchi and the lesion (Fig. 4). Under real time ultrasonic guidance the needle will be placed in the lesion. Suction is applied with a syringe and the needle is moved back and forth inside the lesion as described with EUS-FNA (Figs. 5, 6a-d).

\section{Results}

To date, several papers have been published on this procedure. Krasnik et al. [10] reported on 11 patients in whom 15 lesions were punctured, without complications. The lesions were located as follows: 4 in region 10L, 4 in region 10R, 1 in region $4 \mathrm{~L}, 3$ in region $4 \mathrm{R}, 1$ in region 1,1 in region 7 , and 1 in region $2 \mathrm{R}$. The lesions ranged from $7 \mathrm{~mm}$ to $80 \mathrm{~mm}$. Biopsies obtained through EBUS-FNA showed malignant cells in 13 lesions and benign cells in 2.

Yasufuku et al. [11] published his first experience in a few patients in 2004. In his second trial [12] he examined 70 patients with mediastinal $(n=58)$ and hilar lymph nodes $(n=12)$. The sensitivity, specificity, and accuracy of EBUS-TBNA in distinguishing benign from malignant lymph nodes were $95.7 \%, 100 \%$, and $97.1 \%$, respectively. There were no complications.

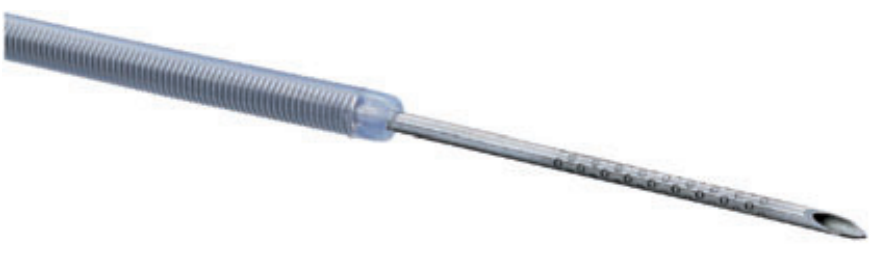

Fig. 3 22-gauge needle.

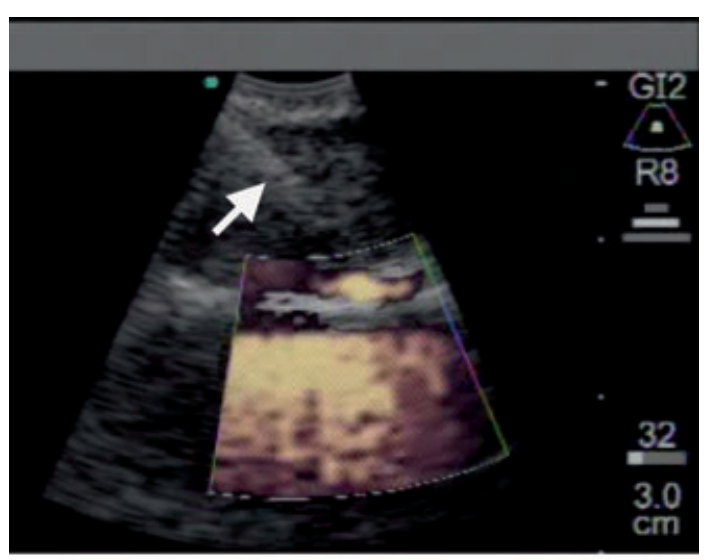

Fig. 4 Control of the vessels during a puncture. The needle is visible (arrow) within the lymphnode.

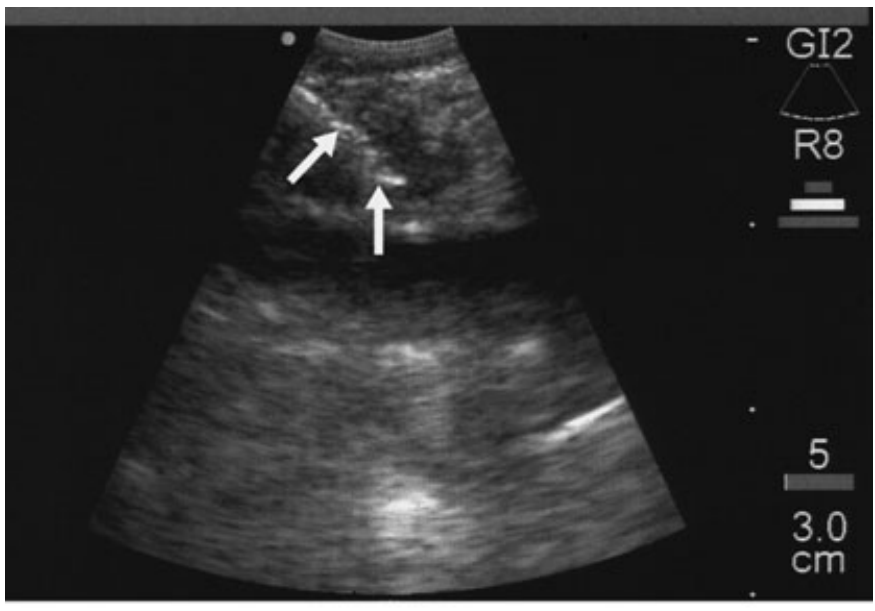

Fig. 5 Puncture of a $8 \mathrm{~mm}$ lymph node in position 4 r. The needle is visible (arrows) within the node.

In a recent paper by Rintoul et al. [13] EBUS-TBNA was used in 18 patients. Cytology revealed node (N)2/N3 disease in 11 patients and provided a primary diagnosis in 8 patients. Cytology results for EBUS-TBNA samples were negative in 6 patients, and mediastinoscopy or clinical follow-up confirmed this result in 4 . Sensitivity, specificity, and accuracy for EBUS-TBNA were $85 \%, 100 \%$, and $89 \%$, respectively.

The largest trial reported the results of the method in 502 patients [14]. 572 lymph nodes were punctured, and 535 (94\%) resulted in a diagnosis. Biopsies were taken from all reachable lymph node stations (2l, 2r, 3, 4r, 4l, 7, 10r, 10l, 11r and $11 \mathrm{l}$. Mean (SD) diameter of the nodes was $1.6 \mathrm{~cm}(0.36 \mathrm{~cm})$ and the range was 0.8 to $3.2 \mathrm{~cm}$. Sensitivity was $92 \%$, specificity was 
a

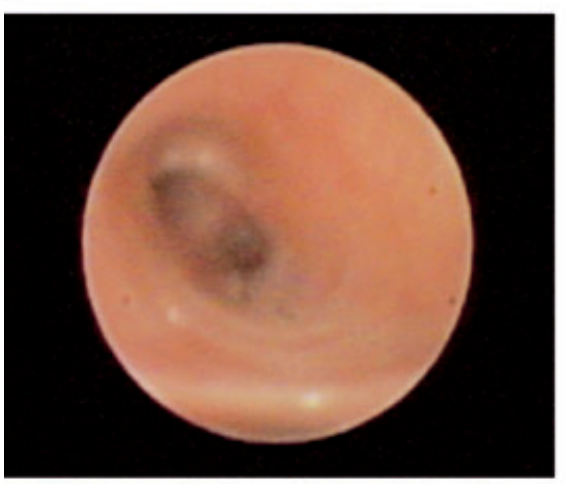

c

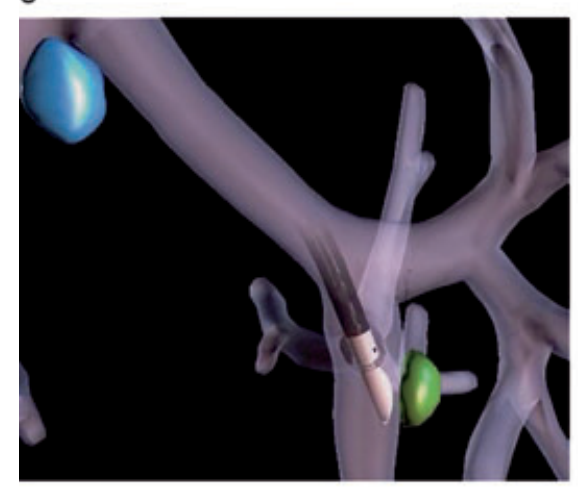

b

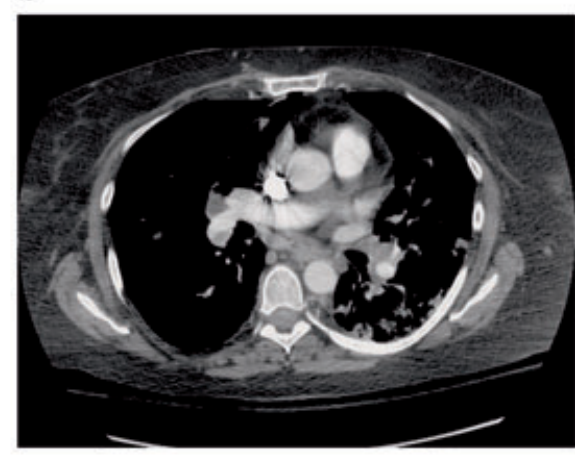

d

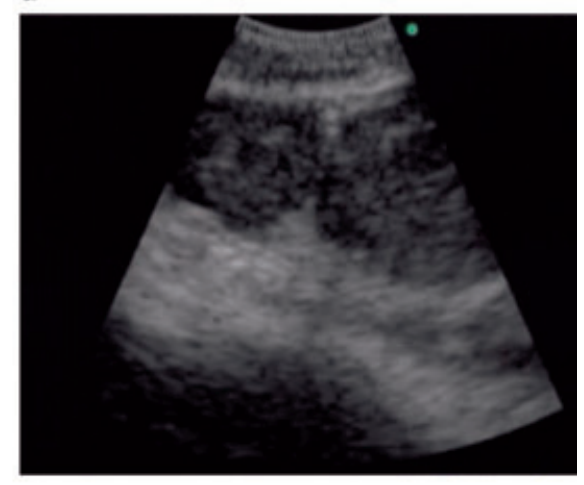

Fig. 6 a-d Enlarged nodes in $11 \mathrm{I}$ in the CT (b) and the EBUS images (c). images a shows the endoscopic view and $c$ and animation. Cytological proof of N3 positive squamous cell cancer.
$100 \%$, and the positive predictive value was $93 \%$. Like in all other trials no complications occurred.

The danish-german group [15] examined in addition the accuracy of EBUS-TBNA in sampling nodes less than $1 \mathrm{~cm}$ in diameter. Among 100 patients 119 lymph nodes with a size between 4 up to $10 \mathrm{~mm}$ were detected and sampled. Malignancy was detected in 19 patients but missed in 2 others; all diagnoses were confirmed by surgical findings. The mean (SD) diameter of the punctured lymph nodes was $8.1 \mathrm{~mm}$. The sensitivity of EBUS-TBNA for detecting malignancy was $92.3 \%$; the specificity was $100 \%$; and the negative predictive value was $96.3 \%$. Again no complications occurred. They summarized, that EBUS-TBNA can sample even small mediastinal nodes, therefore avoiding unnecessary surgical exploration in 1 of 5 patients who have no CT evidence of mediastinal disease. Potentially operable patients with clinically nonmetastatic NSCLC may benefit from presurgical EBUS-TBNA biopsies and staging.

\section{EBUS-TBNA in combination with EUS-FNA:}

Two papers are published about the combined approach. Again Herth et al. [16] could show, that the sensitivity and the specfity of using both techniques obtain the same results as a routine mediastinoscopy.

A recent study [17] has shown promising results regarding the combination of EUS-FNA and EBUS-TBNA. A comparison of EUSFNA and EBUS-TBNA was performed in 33 patients i.e. lung cancer staging in patients with an established diagnosis of NSCLC (20 patients) or diagnosis of a lesion in the mediastinum sus- pected of malignancy in patients suspected of lung cancer (13 patients). The diagnoses could be verified in 28 of 31 patients either by thoracotomy $(\mathrm{n}=9)$ or by clinical follow up $(\mathrm{n}=18)$. A total of 119 lesions were sampled by EUS-FNA $(n=59)$ and EBUS-TBNA $(\mathrm{n}=60)$. EUS-FNA and EBUS-TBNA demonstrated cancer in 26 and 28 lesions respectively, and benign cytology in 30 and 28 lesions respectively. Suspicious cells were found in 3 and 4 lesions by EUS-FNA and EBUS-TBNA, respectively. When comparing the 60 EBUS-TBNA samples with the 59 EUS-FNA samples, 11 additional cancer diagnoses and 3 samples with suspicious cells were obtained by EBUS-TBNA not obtained by EUSFNA. In contrast to this, EUS-FNA diagnosed 12 additional cancer diagnoses, one suspicious and one specific benign diagnosis (sarcoidosis) in addition to EBUS-TBNA. When looking at the results of the combined approach (EUS-FNA+EBUS-TBNA) in 28 of 31 patients in whom a final diagnosis could be obtained, regarding evaluation of cancer involving the mediastinum, 20 patients were found to have mediastinal involvement whereas no mediastinal metastases were found in 8 patients. The accuracy of EUS-FNA and EBUS-TBNA, in combination, for diagnosis of mediastinal cancer was $100 \%$ (95\% CI $83-100 \%$ ). The authors concluded that EUSFNA and EBUS-TBNA seems to be complementary methods.

\section{Conclusion}

Endobronchial ultrasound has been widely available for more than 5 years. A growing body of good-quality literature supports its significant role in airway assessment and procedure guidance. With the EBUS-TBNA scope the next step of development is available. 
The results of the trials demonstrated a high diagnostic rate in the correct prediction of lymph node staging in lung cancer patients compared to other modalities. Moreover, EBUS-TBNA spared many invasive procedures. There were also no complications during all of the procedures. EBUS-TBNA is a minimal invasive procedure with a high diagnostic rate and many patients will benefit from the procedure. EBUS-TBNA should be considered for staging of mediastinal lymph nodes as well as diagnosis of lung cancer.

The combined approach of EUS-FNA and EBUS-TBNA may replace more invasive methods in the evaluation of lung cancer patients suspected of hilar or mediastinal metastases as well as in the evaluation of unknown mediastinal or hilar lesions.

\section{References}

${ }^{1}$ Spira A, Ettinger DS. Multidisciplinary management of lung cancer. $\mathrm{N}$ Engl J Med. 2004; 350: 379-392

${ }^{2}$ Sihoe AD, Yim AP. Lung cancer staging. J Surg Res 2004; 117: 92 - 106

${ }^{3}$ Toloza EM, Harpole L, Detterbeck F, McCrory DC. Invasive staging of non-small cell lung cancer: a review of the current evidence. Chest 2003; 123: $157-166$

${ }^{4}$ Wiersema MJ, Vilmann P, Giovannini M, Chang KJ, Wiersema LM. Endosonography guided fine needle aspiration biopsy: Diagnostic accuracy and complication assessment. Gastroenterology 1997; 112: $1087-1095$

${ }^{5}$ Vilmann P, Hancke S. A new biopsy handle instrument for endoscopic ultrasound guided biopsy. Gastrointest Endosc 1996; 43: 238-242

${ }^{6}$ Hoffmann $\mathrm{H}$. Invasive staging of lung cancer by mediastinoscopy and video-assisted thoracoscopy. Lung Cancer 2001; 34: 3-5

${ }^{7}$ Herth F, Becker HD, Ernst A. Conventional vs Endobronchial Ultrasound-Guided Transbronchial Needle Aspiration: A Randomized Trial. Chest. 2004; 125 (1): $322-325$

${ }^{8}$ Herth F, Becker HD. Endobronchial ultrasound of the airways and the mediastinum. Monaldi Arch Chest Dis 2000; 55: 36-45

${ }^{9}$ Herth F, Hecker E, Hoffmann H, Becker HD. Endobronchial ultrasound for local tumour and lymph node staging in patients with centrally growing lung cancer. Ultraschall Med 2002; 23 (4): 251 - 255

10 Krasnik M, Vilmann P, Larsen SS, Jacobsen GK. Preliminary experience with a new method of endoscopic transbronchial real time ultrasound guided biopsy for diagnosis of mediastinal and hilar lesions. Thorax. 2003; 58 (12): $1083-1086$

11 Yasufuku K, Chhajed PN, Sekine Y, Nakajima T, Chiyo M, Iyoda A, Yoshida S, Otsuji M, Shibuya K, Iizasa T, Saitoh Y, Fujisawa T. Endobronchial ultrasound using a new convex probe: a preliminary study on surgically resected specimens. Oncol Rep. 2004; 11 (2): 293-296

${ }^{12}$ Yasufuku K, Chiyo M, Sekine Y, Chhajed PN, Shibuya K, Iizasa T, Fujisawa T. Real-time Endobronchial Ultrasound-Guided Transbronchial Needle Aspiration of Mediastinal and Hilar Lymph Nodes. Chest 2004; $126: 122-128$

${ }^{13}$ Rintoul RC, Skwarski KM, Murchison JT, Wallace WA, Walker WS, Penman ID. Endobronchial and endoscopic ultrasound-guided real-time fine-needle aspiration for mediastinal staging. Eur Respir J 2005; 25 : 416-421

${ }^{14}$ Herth FJF, Eberhardt R, Vilman P, Krasnik M. EBUS-TBNA - a new device for endoscopic transbronchial real time ultrasound guided biopsy for diagnosis and staging of mediastinal lymphnodes. Thorax, in press

${ }^{15}$ Herth FJF, Krasnik M, Eberhardt R, Ernst A, Vilman P, Dienemann H. EBUS guided biopsy for the staging of mediastinal lymph nodes in a radiologically normal mediastinum. Lung cancer 2005; 49 (2): 26

${ }^{16}$ Herth FJ, Lunn W, Eberhardt R, Becker HD, Ernst A. Transbronchial vs. Transesophageal Ultrasound-guided Aspiration of Enlarged Mediastinal Lymph Nodes. Am J Respir Crit Care Med. 2005; 171: 1164 - 1167

17 Vilmann P, Krasnik M, Larsen SS, Jacobsen GK, Clementsen P. Endoscopic trans-esophageal and Endoscopic-ultrasound guided biopsy: A combined approach in the evaluation of mediastinal lesions. Endoscopy $2005 ; 37$ (9): $833-839$ 